\title{
Energy- Efficient Protocols in Wireless Sensor Networks: A Survey
}

\author{
*Vicky Choudhary, **Mr. Karan Mahajan \\ *Department of Information Technology, Chandigarh group of College, Landran, Mohali \\ **Department of Information Technology, Chandigarh group of College, Landran, Mohali
}

\begin{abstract}
A wireless sensor network consist sensor nodes of large number can be used as an effective tool for gathering data in various situations. One of the major issues in WSN is the development of a routing protocol which is energy-efficient and has a significant impact on the overall lifetime of the sensor network [1]. The vital issue in designing the wireless sensor networks is Energy saving. The main significant issue is the battery power which is limited inherent within sensor nodes of networks. Therefore in order to enhance the life time of the network. it is necessary to design effective and energy aware protocols. In this paper, a review on structure of network routing based protocol is a main type which consist hierarchical based routing protocol as subtype in WSNs is carried out.
\end{abstract}

Keywords:- Wireless, Sensor network, Energy consumption, energy efficient, network life time, routing protocols, sensor, battery, life.

\section{INTRODUCTION}

A WSN is an enlarged wireless network which consists of a maximum number of sensors and one base station. These sensor nodes are small devices that consist of four basic components 1) sensing subsystem, 2) processing subsystem, 3) wireless communication subsystem 4) energy supply subsystem. In most cases, the sensors forming these networks are deployed randomly and left unattended to and are expected to perform their mission properly and efficiently. A real and appropriate solution for this problem is to implement routing protocols that perform efficiently and utilizing the less amount of energy as possible for the communication among nodes.

Therefore, one good approach is to consider that sensors located in the same region of the network will transmit similar values of the attributes. Sensor networks need protocols, which are specific, data centric, capable of aggregating data and optimizing energy consumption. Sensor nodes deployed in a specified area monitors environmental conditions such as temperature, air pressure, humidity, light, motion or vibration, and so on. The sensor nodes are usually programmed to monitor or collect data from surrounding environment and pass the information to the base station for remote user access through various communication technologies.

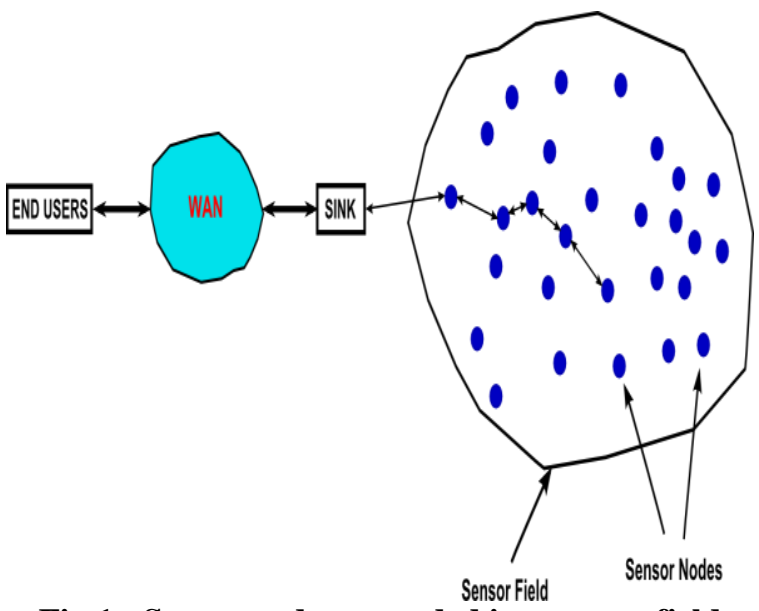

Fig 1: Sensor nodes spreaded in a sensor field

\section{ROUTING PROTOCOLS}

A WSN can have structure of network based or protocol operation routing based protocol . Depending on the application routing protocols in WSNs may differ (Protocol-Operation-based) and network architecture 
(Network-Structure-based) as shown in Fig. 2. Based on the underlying network there are three protocol categories:

\section{A. Flat Routing}

Each node plays the same role and sensor nodes collaborate to perform the sensing task.

\section{B. Hierarchical Routing}

Hierarchical routing is an efficient way to lower energy consumption within a cluster, performing data aggregation and fusion in order to decrease the number of transmitted messages to the sink node.

\section{Location-based}

Sensor nodes are addressed by means of their locations. On the basis of strengths of incoming signal the distance between neighboring nodes can be estimated. Relative coordinates of neighboring nodes can be obtained by exchanging such information between neighbors or by communicating with a satellite using GPS. To save energy, some location-based schemes demand that nodes should go to sleep if there is no activity.

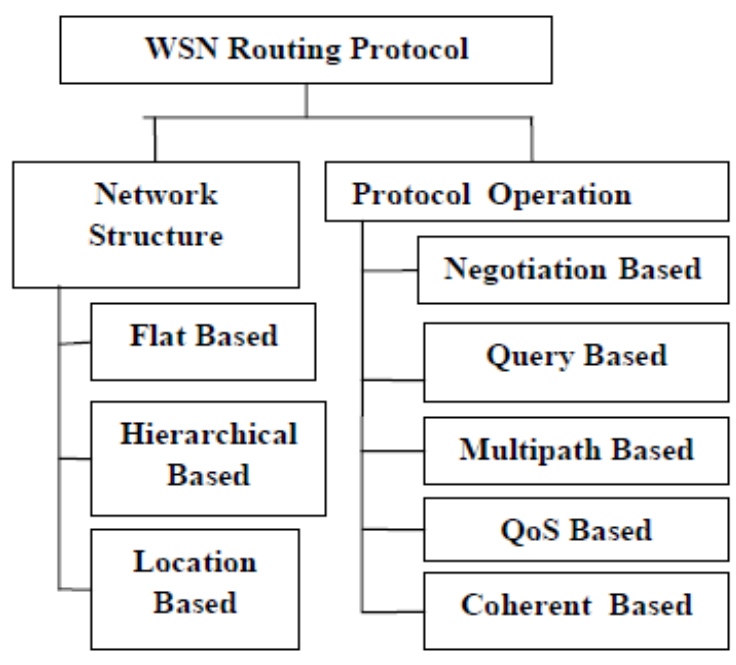

Fig.2 Classification of WSN Routing Protocols

Depending on the Protocol Operation we can divide routing protocols in:

\section{Multipath-based}

They use multiple paths rather than a single path in order to enhance network performance. For instance the fault tolerance can be increased by maintaining multiple paths between the source and destination at the expense of increased energy consumption and traffic generation.

\section{Query-based}

The destination nodes propagate a query for data from a node through the network; a node with this data sends the data that matches the query back to the node that initiated it.

\section{Negotiation-based}

Use negotiation in order to eliminate redundant data transmissions. Communication decisions are also made based on the resources available.

\section{QoS-based}

When delivering data, the network balances between energy consumption and data quality through certain QoS metrics as delay, energy or bandwidth.

\section{Coherent-based}

The entity of local data processing on the nodes distinguish between coherent (minimum processing) and noncoherent (full processing) routing protocols.

\section{HIERARCHICAL PROTOCOLS}

In this paper various hierarchical based routing protocols has been reviewed. A hierarchical approach breaks the network into clustered layers. Nodes are grouped into clusters consist of a cluster head which is responsible for routing from the cluster to the various other cluster heads /base stations. Data travels from a lower clustered layer to a higher one. The most popular energy-efficient hierarchical clustering algorithm for WSNs is Low-energy adaptive clustering hierarchy (LEACH) LEACH [4] that was proposed for reducing power consumption. LEACH is based on an aggregation technique that combines the original data into a smaller 
size of data that carry only meaningful information to all individual sensors. LEACH divides the network into several clusters of sensors, which are constructed by using localized coordination and control. LEACH uses a randomize rotation of high-energy $\mathrm{CH}$ position rather than selecting in static manner, to give a chance to all sensors to act as CHs and avoid the battery depletion of an individual sensor and die quickly. Therefore, it is not suitable to networks when used in enlarged regions. While LEACH helps the sensors within their cluster dissipate their energy slowly, the $\mathrm{CHs}$ consume a larger number of energy when located ahead from the sink.

Fig. 3 LEACH operations

The major characteristics of this Protocol are as follow:

-It rotates the cluster heads in a randomized fashion to achieve balanced energy consumption,

-Sensors have synchronized clocks so that they know the beginning of a new cycle,

-Sensors do not need to know location or distance information.

There are some drawbacks of this protocol such as:

-LEACH uses single-hop routing where each node can transmit directly to the cluster-head and the sink. Therefore, it is not suitable to networks used in large regions.

-The thought of dynamic clustering brings additional overhead, e.g. head changes, advertisements etc., which may decrease the gain in energy consumption.

-Random election of $\mathrm{CH}$, hence there is Possibility that all $\mathrm{CHs}$ will be concentrated in same area.

-The protocol thinks that all nodes start with the same number of energy capacity in each round. Of election

This protocol is most suited for constant monitoring such as monitor machinery for fault detection and diagnosis.

\section{Power-Efficient Gathering in Sensor Information Systems (PEGASIS):}

PEGASIS is an development of the LEACH protocol, which forms sequence from sensor nodes so that each node send and receives from a neighbor and only one node is chosen from that sequence to send to the base station (sink). The data is gathered and moves from node to node, aggregated and eventually sent to the base station. PEGASIS [7] avoids formation of cluster and in a sequence uses only one node to send to the BS (sink) instead of using multiple nodes. A sensor transmits to its local neighbors in the data fusion phase instead of sending directly to its $\mathrm{CH}$ as in the case of LEACH. When a sensor fails or dies due to low battery power, the chain is constructed using the same greedy approach by bypassing the failed sensor.

\section{Hierarchical PEGASIS:}

An extension to PEGASIS, called Hierarchical-PEGASIS was introduced with the objective of decreasing the delay incurred for packets during transmission to Base Station the BS. H-PEGASIS proposes a solution to the data gathering problem by considering energy $\mathrm{x}$ delay metric.

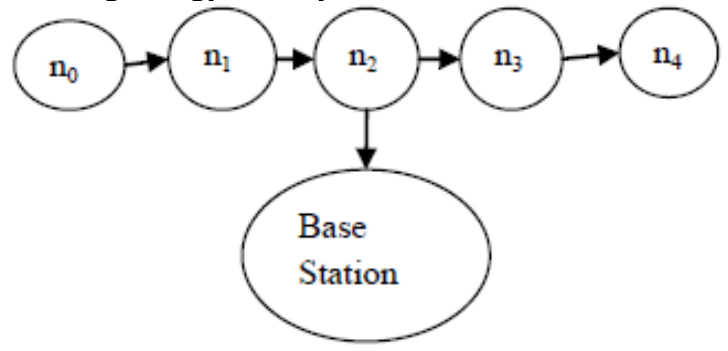

Fig.3 Chaining in PEGASIS

\section{Energy Balancing PEGASIS (EB-PEGASIS):}

EBPEGASIS is an energy efficient chaining algorithm in which a node will consider average distance of formed chain. If the distance from closest node to its upstream node is longer than distance- threshold, the closest node is a far node. In this condition, the far node will search a nearer node on formed chain. Through this method, the new protocol EB-PEGASIS can avoid long chain effectively. It not only saves energy on sensors, but also balances the energy consumption of all sensor nodes.

\section{Hybrid, Energy-Efficient Distributed Clustering (HEED):}

HEED operates in multi-hop networks, using an flexible power transmission in the inter-clustering communication. HEED was introduced with four main goals.(i) prolonging network lifetime by distributing 
energy consumption (ii) terminating the process of clustering within a stable number of iterations (iii) minimizing control overhead, and (iv) Producing well distributed CHs and compact clusters. In HEED, the recommended algorithm regularly selects $\mathrm{CHs}$ conforming to the mixture of two clustering parameters. Each sensor node has their residual energy is the main parameter and as a function of cluster density or node degree (i.e. amount of neighbors) is intra-cluster communication cost is the secondary parameter. HEED clustering improves network lifetime over LEACH clustering because LEACH randomly selects $\mathrm{CHs,} \mathrm{which} \mathrm{may} \mathrm{result}$ some nodes in rapid death

\section{The important features of this protocol are as follows:}

-HEED distribution of energy extends the lifetime of the nodes within the network thus stabilizing the neighboring node.

-HEED does not require special node capabilities, such as location-awareness

-HEED does not make assumptions about node distribution. The nodes also automatically update their neighbor sets in multi-hop networks by periodically sending and receiving messages. It operates correctly even when nodes are not synchronized.

\section{Threshold Sensitive Energy Efficient Sensor Network Protocol (TEEN):}

TEEN is a clustering protocol i.e. hierarchical, which assembles sensors into clusters each which is accompanied by $\mathrm{CH}$. The sensed data is reported to their $\mathrm{CH}$ by the sensors within a cluster. The higher level $\mathrm{CH}$ receives combined data from $\mathrm{CH}$ until the data reaches the sink. Thus, TEEN consist of the sensor network architecture is based on a hierarchical assembling in which closer nodes form clusters in first level and continues to the second level until the BS (sink) is extended. TEEN uses hierarchical approach with data-centric method.

\section{The main features of this protocol are as follows:}

-Time critical data reaches the user almost instantaneously.

-The soft threshold can be varied, depending on the criticality of the sensed attribute and the target application.

-A smaller value of the soft threshold gives a more accurate picture of the network, at the expense of increased energy consumption.

-At every cluster change time, the attributes are broadcast afresh and so, the user can change them as required.

\section{CONCLUSION}

One of the main challenges in the design of routing protocols for WSNs is energy efficiency due to the scarce energy resources of sensors. The energy consumption of the sensors is dominated by data transmission and reception. Therefore, routing protocols designed for WSNs should be as energy efficient as possible to prolong the lifetime of individual sensors, and hence the network lifetime. The protocols discussed have individual advantages and pitfalls. For realization of sensor networks, it is needed to satisfy the constraints that is introduced by various factors example cost, fault tolerance, scalability, topology change, environment, and power consumption. In this paper, hierarchical based routing protocols are discussed on the basis of network structure. Many issues and challenges still exist that need to be solved in the sensor networks.

\section{REFERENCES}

[1] K. Padmanabhan 1, Dr. P. Kamalakkannan 2 "Energy Efficient Adaptive Protocol for Clustered Wireless Sensor Networks" IJCSI International Journal of Computer Science Issues, Vol. 8, Issue 5, No 1, September 2011 ISSN (Online): 1694-0814

[2] Network" IEEE International Conference on pp.393to398,December2008.Rajashree.V.Biradar, Dr. S. R. Sawant Dr. R. R. Mudholkar ,Dr. V.C.Patil Multihop” Routing In Self-Organizing

[3] Wireless Sensor Networks" IJCSI International Journal of Computer Science Issues, Vol. 8, Issue 1, January 2011.W.R. Heinzelman, A. Chandrakasan, and H. Balakrishnan, "Energy-Efficient Communication Protocol for Wireless

[4] Microsensor Networks",Proceedings of the 33rd Hawaii International Conference on System Sciences, 2000.S.Hussain, and O. Islam,An Energy Efficient Spanning Tree Based Multi-Hop Routing in Wireless Sensor

[5] Networks," Proceedings of Wireless Communications and Networking Conference, pp. 43834388,2007.S.Lindsey, C. S.Raghavendra, and K. M. Sivalingam, " Data Gathering Algorithms in Sensor Networks Using Energy Metrics,” IEEE Transactions on Parallel Distributed System, Vol. 13, Issue. 9, pp. 924-935, 2002.

[6] Osama Younis et.al'Distributed Clustering in Ad-hoc Sensor Networks: A Hybrid, Energy-Efficient Approach", Proceedings of IEEE INFOCOM, Hong Kong, an extended version appeared in IEEE Transactions on Mobile Computing, 2004. 
[7] S.M. Jung, Y. J. Han, and T. M. Chung, “ The Concentric Clustering Scheme for Efficient Energy Consumption in the PEGASIS, on Advanced Communication Technology, Vol. 1, pp. 260-2 Proceedings of the 9th International Conference65, 2007.

[8] Xiaoli Li, Hong chi Shi, and Yi Shang," A Sorted RSSI Quantization Based Algorithm for Sensor Network Localization" Proceedings of the 2005 11th International Conference on Parallel and Distributed Systems (ICPADS'05)

[9] The Math Work - MATLAB and Stimulant for technical Computer 